\title{
Suitability of AFLP and Microsatellite Marker Analysis for Discriminating Intraclonal Variants of the Potato Cultivar Russet Norkotah
}

\author{
Anna L. Hale ${ }^{1}$ and J. Creighton Miller, Jr. ${ }^{2}$ \\ Department of Horticultural Sciences, Texas A\&M University, College Station, TX 77843-2133 \\ K. Renganayaki and Alan K. Fritz ${ }^{3}$ \\ Department of Soil and Crop Sciences, Texas A\&M University, College Station, TX 77843-2474 \\ J.J. Coombs, L.M. Frank, and D.S. Douches \\ Department of Crop and Soil Sciences, Michigan State University, East Lansing, MI 48824-1325
}

\begin{abstract}
AdDitional INDEX wORDS. Solanum tuberosum, subclonal variants, DNA fingerprinting, SSR
ABstract. The objective of this study was to differentiate six intraclonal variants of the potato (Solanum tuberosum $\mathrm{L}$.) cultivar Russet Norkotah. One-hundred-twelve AFLP primer combinations producing 3755 bands and 79 microsatellite primers producing over $\mathbf{4 0 0}$ bands failed to identify any reproducible polymorphisms among the intraclonal variants and 'Russet Norkotah'. The inability to detect differences between clones underscores the degree of genetic similarity between them, despite differences in phenotypic expression. This inability could be due to the tetraploid nature of the clones and/or to epigenetic differences not detected by the utilized procedures.
\end{abstract}

The potato cultivar Russet Norkotah was released in 1987, and it quickly became the early market cultivar of choice in the national marketplace (Johansen et al., 1988). By 1997, due in part to wide promotion, 'Russet Norkotah'had become the second most popular cultivar in the United States, with over 23,000 acres of certified seed grown in the United States and Canada (National Potato Council, 1998). The popularity of 'Russet Norkotah' is largely attributed to factors such as attractive tubers, tuber uniformity, resistance to hollow heart and second growth, good storability, and a high percentage of marketable tubers. While possessing mostly positive features, 'Russet Norkotah' does have some negative characteristics such as susceptibility to potato virus Y (PVY) and verticillium wilt (Verticillium dahliae Kleb.), weak vines, and requirements for large inputs of nitrogen fertilizer and pesticides. Although 'Russet Norkotah' was in high demand in the potato industry, its often weak vines and other negative characteristics made it unsuitable for profitable growing in Texas and other early-market production areas. If these production areas were to remain competitive in supplying 'Russet Norkotah' tubers to the market, it became clear that improved strains with stronger vines would be required.

Previous breeding and selection efforts had proven that potato cultivars can be improved through strain selection. Notable successes include 'Russet Burbank' from 'Burbank' (Miller, 1954), 'Red LaSoda' from 'LaSoda' (Miller, 1954), 'Dark Red Norland' from 'Norland,' and 'Norgold Russet Strain M' from 'Norgold Russet' (Miller et al., 1995). Following the example set by the success with 'Norgold Russet', the Texas Potato Variety Development Program began to make selections and evaluate improved

Received for publication 14 Jan. 2005. Accepted for publication $29 \mathrm{Jan}$. 2005. This work is part of the Dissertation of Anna L. Hale for a PhD degree in genetics.

${ }^{1}$ Current address: USDA, ARS, U.S. Vegetable Laboratory, 2700 Savannah Highway, Charleston, SC 29414-4715.

2Corresponding author; email: jcmillerjr@tamu.edu.

${ }^{3}$ Current address Dept. of Agronomy, Kansas State Univ., 2004 Throckmorton Hall, Manhattan, KS 66506. strains of 'Russet Norkotah'(Miller et al., 1999). Two years later, a similar selection project was initiated by the Colorado State Univ. Potato Breeding Program. By 1998, a 9-year selection project, based primarily on yield, vine size, and tuber type had produced eight promising intraclonal selections of 'Russet Norkotah'. These were TXNS 102, TXNS 112, TXNS 223, TXNS 249, TXNS 278, TXNS 296, CORN 3, and CORN 8. Six of these, TXNS 112, TXNS 223, TXNS 278, TXNS 296, CORN 3, and CORN 8, have been granted Plant Variety Protection (PVP). By 1999, 38\% of the 'Russet Norkotah' acreage entered into seed certification was to one of these five strains (National Potato Council, 2000), with acreage of the strains increasing every year. By 2001, $42 \%$ of the U.S. 'Russet Norkotah' acreage entered into certification was planted to the strains (National Potato Council, 2002), and by 2002, over half (52\%) (National Potato Council, 2003). The intraclonal selections from both the Texas and Colorado programs exhibit higher yield, lower nitrogen requirements, later maturity, longer flowering periods, and generally larger tuber size than standard 'Russet Norkotah' (Miller et al., 1999; Zvomuya et al., 2002)

Despite several quantitative differences (e.g., vine size, maturity, average tuber size, yield, etc.) among the strains and standard 'Russet Norkotah', it can be difficult to distinguish them from one another. Although 'Russet Norkotah' intraclonal selections have been discriminated using canonical and cluster analysis (Miller et al., 2004), molecular markers might be especially helpful in distinguishing these selections and aid in the granting of PVP in the United States and/or Plant Breeder's rights in Canada for additional new strain selections. Furthermore, markers that could differentiate strains would be useful for certification and help to deter infringement of patent rights in the future.

Previous research has demonstrated that both the AFLPand microsatellite approaches are promising for differentiating between cultivars, and potentially, somatic mutants. AFLPs have a high multiplex ratio, thus generate volumes of data, while SSRs are highly polymorphic and have been successful in discriminating between closely related species. For example, Cabrita et al. (2001) were able to distinguish between field-selected clonal variants 
of fig (Ficus carica L.) using AFLP analysis, and Vignani et al. (1996) found polymorphisms between seven probable somatic mutants of the grape (Vitis vinifera L.) cultivar Sangiovese using SSR analysis. Several successful studies have been conducted on potato using AFLP and SSR analysis (Kardolus et al., 1998; Kim et al., 1998; McGregor et al., 2000; Meksem, 1995; Meyer et al., 1998; Milbourne et al., 1997, 1998; Provan et al., 1996; Raker and Spooner, 2002; Schneider and Douches, 1997). Thus, a multifaceted approach using the aforementioned techniques could reasonably be employed to distinguish between the 'Russet Norkotah' clonal selections.

The primary objective of this study was to identify polymorphisms among six intraclonal variants of the potato cultivar Russet Norkotah using AFLPs and SSRs. Polymorphisms discovered would provide additional evidence in supporting, granting, and enforcing individual plant variety protection to new additional strain selections.

\section{Materials and Methods}

Plant material. Six clonal selections (TXNS 112, TXNS 223, TXNS 278, TXNS 296, CORN 3, and CORN 8), standard 'Russet Norkotah', and a white-flesh chipping Texas breeding line, ATX85404-8W, were subjected to AFLP and SSR analysis. ATX85404-8W was included as a check since it was expected to differ significantly from 'Russet Norkotah'. For the SSR analysis, 'Russet Burbank' was used as the check and TXNS 102 was included as well.

DNA EXtraction. DNA was isolated from leaves of potato plants grown near Springlake, Tex., in Spring 1999 using a procedure developed by Fulton et al. (1995). The DNA concentration of each genotype was determined based on the absorbance at 260 $\mathrm{nm}$. Following the determination of concentration, each DNA sample was diluted to a concentration of $50 \mathrm{ng} \cdot \mu \mathrm{L}^{-1}$. The same samples were used for AFLP and microsatellite analysis.

AFLP ANALYsis I. AFLP reactions were conducted following the method described by Renganayaki et al. (2001). Selective amplification was performed using all 64 combinations of EcoRI and $M s e I$ primers with three selective nucleotides provided with the AFLPAnalysis System I kit (Life Technologies, Gaithersburg, Md.). The PCR products were separated on a $5 \%$ denaturing polyacrylamide gel, and the DNA bands were visualized using silver staining (Fritz et al., 1999). Gels were scored visually for the presence or absence of bands.

AFLP ANALYsis II. The DNA from each of the genotypes was sequentially digested with $\mathrm{Mse}$ I and PstI and ligated to adapters according to Menz et al. (2002). The restricted adapter-ligated DNA was diluted to $1 \mathrm{ng} \cdot \mu \mathrm{L}^{-1}$ with water. The resulting mixture was termed restricted, adapter-ligated, diluted DNA (RAD).

After optimizing the $\mathrm{MgCl}_{2}$ concentration, preamplification was performed according to Klein et al. (2000) with minor modifications. Twenty-five-microliter reactions were performed using $5-\mu \mathrm{L}$ RAD template, 1X PCR buffer, $1.5 \mathrm{~mm} \mathrm{MgCl}_{2}, 160 \mu \mathrm{M}$ dNTPs, 0.2 U Taq DNA polymerase (Promega Corp., Madison, Wis.), $0.56 \mu \mathrm{M} \mathrm{Mse} \mathrm{I}+0$ pre-amp primer, and $0.56 \mu \mathrm{M} P s t \mathrm{I}+0$ pre-amp primer. Sequence of the primers is shown in Table 2. The PCR profile was identical to that of Klein et al. (2000), except 25, as opposed to 20 , cycles were performed. The resulting product was diluted 1:10 with water, and run on a 1\% agarose gel to verify amplification.

Following preamplification, selective amplification was performed using primers with three selective bases on the 3 ' end. For visualization purposes, the $P s t \mathrm{I}$ selective primer was labeled with IRD dye (LI-COR Biosciences, Lincoln, Nebr.). Ten-microliter selective amplification reactions contained $2 \mu \mathrm{L}$ dilute preamplified DNA, 1X PCR buffer, $1.25 \mathrm{~mm} \mathrm{MgCl}_{2}, 200 \mu \mathrm{M}$ dNTPs, 0.2 U Taq DNA polymerase (Promega), $0.25 \mathrm{pm} \mathrm{MseI} \mathrm{primer,}$ and $15 \mathrm{~nm}$ PstI Primer (IRD labeled). The PCR reaction was run under the following profile. Cycle one began with a 2-min hold at $95{ }^{\circ} \mathrm{C}$ followed by continued denaturing for $1 \mathrm{~min}$ at $94{ }^{\circ} \mathrm{C}$, a 1 -min annealing step at $65^{\circ} \mathrm{C}$, and a $1-$ min-30-s extension at 72 ${ }^{\circ} \mathrm{C}$. The annealing step was reduced by $0.6^{\circ} \mathrm{C}$ each cycle for the next 12 cycles, giving a touch down phase of 13 cycles. Twentythree cycles were then performed with an annealing temperature of $56^{\circ} \mathrm{C}$. Following these 23 cycles, an additional extension step at $72{ }^{\circ} \mathrm{C}$ for 5 min was performed.

PstI primers with three different selective nucleotides (CGT, CTT, and CAA) were used. CTT and CGT were labeled with IRDye TM 800, and CAA was labeled with IRDye TM 700. Thus, it was possible to run two different IRD-labeled Pst I primers, one visualized at $700 \mathrm{~nm}$, and one visualized at 800 $\mathrm{nm}$, simultaneously in each well. Using different wavelengths on primer labels allowed the running of twice as many primer pairs per gel as was previously possible. The same 16 unlabeled MseI primers used in the silver staining analysis were used for the LI-COR analysis. Each of these 16 primers were run with each of the three Pst I primers, producing a grand total of 48 different primer combinations used in the selective amplification step for the LI-COR analysis (Table 2).

The AFLP amplification products were analyzed using a LICOR model 4200 dual-dye automated DNA sequencing system according to Klein et al. (2000). Each sample ( $1 \mu \mathrm{L})$ was loaded on a $6 \%$ Long Ranger (Cambrex Corp., East Rutherford, N.J.) polyacrylamide gel containing $7 \mathrm{M}$ urea, when lower bands were not separated satisfactorily, a 7\% Long Ranger polyacrylamide gel was used. Gels were scored visually for presence or absence of bands.

Microsatellite ANALYSIS. In addition to AFLPs, microsatellite markers were used. Seventy-nine potato microsatellite primers pairs, originally identified by Milbourne et al. (1998) were used in this analysis (Table 1) and reaction conditions were modified from Raker and Spooner (2002). Conditions for a $25-\mu \mathrm{L}$ reaction were as follows: 1X PCR Buffer (Sigma-Aldrich, St. Louis), $1.5 \mathrm{~mm} \mathrm{MgCl}_{2}, 0.2 \mathrm{~mm}$ dNTPs (Gibco BRL, Life Technologies, Gaithersberg, Md.), $0.4 \mu \mathrm{M}$ of each unlabeled primer pair (SigmaGenosys, The Woodlands, Tex.), 1U DNA polymerase (REDTaq; Sigma-Aldrich), and $20 \mathrm{ng}$ DNA. The PCR profile followed was identical to that of Raker and Spooner (2002).

Reaction products were dried in a speed vac (Savant, Holbrook, N.Y.), at medium speed until completion, and were resuspended in $5 \mu \mathrm{L}$ of water and $5 \mu \mathrm{L}$ of formamide manual sequencing dye (Sambrook et al., 1989). The resulting mixture was denatured for $3 \mathrm{~min}$ at $90{ }^{\circ} \mathrm{C}$ prior to loading. The denatured products were separated on a $6 \%$ denaturing polyacrylamide gel, and the DNA bands were visualized using silver staining (Fritz et al., 1999). Gels were scored visually for the presence or absence of bands.

\section{Results}

AFLP AnAlysis. A total of 112 AFLP primer combinations was used in this study, including 64 EcoRI/MseI primer pairs, which amplified both methylated and nonmethylated regions, and the 48 PstI/MseI primer pairs, which amplified only nonmethylated regions. A representative gel is shown in Fig. 1. None of the 112 
Table 1. Primer sequences of microsatellite primers used in the analysis of six clonal selections of the potato cultivar Russet Norkotah.

\begin{tabular}{|c|c|c|c|c|c|c|c|}
\hline Primer & & Primer sequence $^{z} 5^{\prime}-3^{\prime}$ & $\begin{array}{l}\text { Melting } \\
\text { temp }\left({ }^{\circ} \mathrm{C}\right)\end{array}$ & Primer & & Primer sequence $^{z} 5^{\prime}-3^{\prime}$ & $\begin{array}{l}\text { Melting } \\
\text { temp }\left({ }^{\circ} \mathrm{C}\right)\end{array}$ \\
\hline STM0003 & F & GGA GAA TCA TAA CAA CCA G & 48 & STM1020 & $\mathrm{R}$ & CCC AAG ATT ACC ACA TTC & 51 \\
\hline STM0003 & $\mathrm{R}$ & AAT TGT AAC TCT GTG TGT GTG & 48 & STM1021 & $\mathrm{F}$ & GGA GTC AAA GTT TGC TCA CAT C & 59 \\
\hline STM0007 & $\mathrm{F}$ & GGA CAA GCT GTG AAG TTT AT & 52 & STM1021 & $\mathrm{R}$ & CAC CCT CAA CCC CCA TAT C & 60 \\
\hline STM0007 & $\mathrm{R}$ & AAT TGA GAA AGA GTG TGT GTG & 52 & STM1030 & $\mathrm{F}$ & GTT CAT TCG GAT AGA CTT GAG ACA & 60 \\
\hline STM0014 & $\mathrm{F}$ & CAG TCT TCA GCC CAT AGG & 53 & STM1030 & $\mathrm{R}$ & TGC AAA TAC TCT AGA GCA AGA AGG & 59 \\
\hline STM0014 & $\mathrm{R}$ & TAA ACA ATG GTA GAC AAG ACA AA & 53 & STM1031 & $\mathrm{F}$ & TGT GTT TGT TTT TCT GTA T & 46 \\
\hline STM0051 & $\mathrm{F}$ & TAC ATA CAT ACA CAC ACG CG & 53 & STM1031 & $\mathrm{R}$ & AAT TCT ATC CTC ATC TCT A & 43 \\
\hline STM0051 & $\mathrm{R}$ & CTG CAA CTT ATA GCC TCC A & 53 & STM1040 & $\mathrm{F}$ & AGT ACT CAG TCA ATC AAA G & 44 \\
\hline STM1005 & $\mathrm{F}$ & ATG CCT CTT ACG AAT AAC TCG G & 59 & STM1040 & $\mathrm{R}$ & AGG TAA GTA TGT TCT CCA G & 46 \\
\hline STM1005 & $\mathrm{R}$ & CAG CTA ACG TGG TTG GGG & 59 & STM1049 & $\mathrm{F}$ & CTA CCA GTT TGT TGA TTG TGG TG & 60 \\
\hline STM1017 & $\mathrm{F}$ & GAC ACG TTC ACC ATA AA & 48 & STM1049 & $\mathrm{R}$ & AGG GAC TTT AAT TTG TTG GAC G & 59 \\
\hline STM1017 & $\mathrm{R}$ & AGA AGA ATA GCA AAG CAA & 48 & STM1051 & $\mathrm{F}$ & TCC CCT TGG CAT TTT CTT CTC C & 66 \\
\hline STM1024 & $\mathrm{F}$ & ATA CAG GAC CTT AAT TTC CCC AA & 59 & STM1051 & $\mathrm{R}$ & TTT AGG GTG GGG TGA GGT TGG & 65 \\
\hline STM1024 & $\mathrm{R}$ & TCA AAA CCC AAT TCA ATC AAA TC & 59 & STM1052 & $\mathrm{F}$ & CAA TTT CGT TTT TTC ATG TGA CAC & 61 \\
\hline STM1029 & $\mathrm{F}$ & AGG TTC ACT CAC AAT CAA AGC A & 58 & STM1052 & $\mathrm{R}$ & ATG GCG TAA TTT GAT TTA ATA CGT AA & 59 \\
\hline STM1029 & $\mathrm{R}$ & AAG ATT TCC AAG AAA TTT GAG GG & 58 & STM1053 & $\mathrm{F}$ & TCT CCC CAT CTT AAT GTT TC & 54 \\
\hline STM1041 & $\mathrm{F}$ & GTT GAG TAG AAG GAG GAT T & 53 & STM1053 & $\mathrm{R}$ & CAA CAC AGC ATS CAG ATC ATC & 54 \\
\hline STM1041 & $\mathrm{R}$ & CCT TTG TCT TCT GCT TTT G & 53 & STM1072 & $\mathrm{F}$ & CAT TAC CTC CAT TGC TA & 47 \\
\hline STM2022 & $\mathrm{F}$ & GCG TCA GCG ATT TCA GTA CTA & 57 & STM1072 & $\mathrm{R}$ & GTC TCG TAC TTC TTC AT & 41 \\
\hline STM2022 & $\mathrm{R}$ & TTC AGT CAA CTC CTG TTG CG & 57 & STM1088 & $\mathrm{F}$ & TGG GGC TTC TTT GAG & 51 \\
\hline STM3009 & $\mathrm{F}$ & TCA GCT GAA CGA CCA CTG TTC & 63 & STM1088 & $\mathrm{R}$ & TCC CAT GGT TCA CCA & 52 \\
\hline STM3009 & $\mathrm{R}$ & GAT TTC ACC AAG CAT GGA AGT C & 63 & STM1091 & $\mathrm{F}$ & GGA CAA TCC ATA AAT CTC AG & 56 \\
\hline STM0001 & $\mathrm{F}$ & AGT ATT CAA CCC ATT GAC TTG GA & 60 & STM1091 & $\mathrm{R}$ & AGC AGC AGC AGC CTC TCC TA & 67 \\
\hline STM0001 & $\mathrm{R}$ & TAG ACA AGC CAA GCT GGA GAA & 60 & STM1092 & $\mathrm{F}$ & AGC TAT GTT AGG GTC AAG GGC & 60 \\
\hline STM0004 & $\mathrm{F}$ & CGA GGG CGT AAA CTC ATG ATA & 60 & STM1092 & $\mathrm{R}$ & CAA GGA TTA AAA GCA CCT C & 53 \\
\hline STM0004 & $\mathrm{R}$ & AGG TTA TTG TGG ACA CAG TCT TCA & 60 & STM1093 & $\mathrm{F}$ & TGT ACT GGG GAG CCT CAA AG & 60 \\
\hline STM0006 & $\mathrm{F}$ & GAA GTT GAC ATT GAG CCC & 54 & STM1093 & $\mathrm{R}$ & AAT TTT AAC CTC GTG ACA TGG G & 60 \\
\hline STM0006 & $\mathrm{R}$ & GGA TAT CCA TTC TTA GAT GCA & 54 & STM1104 & $\mathrm{F}$ & TGA TTC TCT TGC CTA CTG TAA TCG & 60 \\
\hline STM0010 & $\mathrm{F}$ & TCC TTA TAT GGA GCA AGC A & 54 & STM1104 & $\mathrm{R}$ & CAA AGT GGT GTG AAG CTG TGA & 60 \\
\hline STM0010 & $\mathrm{R}$ & CCA GTA GAT AAG TCA TCC CA & 53 & STM1105 & $\mathrm{F}$ & AAA CCT GCT ACA AAT AAG GC & 54 \\
\hline STM0013 & $\mathrm{F}$ & AAC TAT CAA CTA AAT GCC TTT TT & 54 & STM1105 & $\mathrm{R}$ & CAG AAA TAA TTG GAG GAG ATG & 54 \\
\hline STM0013 & $\mathrm{R}$ & TAG ACA AGC CAA GCT GGA GAA & 60 & STM1106 & $\mathrm{F}$ & TCC AGC TGA TTG GTT AGG TTG & 60 \\
\hline STM0017 & $\mathrm{F}$ & ATC ATG ATG ACA CCT ACT ATA ACC & 54 & STM1106 & $\mathrm{R}$ & ATG CGA ATC TAC TCG TCA TGG & 60 \\
\hline STM0017 & $\mathrm{R}$ & TCC ACA CCT CTA TCT GTT GA & 54 & STM2004 & $\mathrm{F}$ & ACC AGC GCA ATA ACT CCG & 60 \\
\hline STM0019 & $\mathrm{F}$ & AAT AGG TGT ACT GAC TCT CAA TG & 53 & STM2004 & $\mathrm{R}$ & GCT TTG TGG ATT CAC CGG & 60 \\
\hline STM0019 & $\mathrm{R}$ & TTG AAG TAA AAG TCC TAG TAT GTG & 53 & STM2005 & $\mathrm{F}$ & TTT AAG TTC TCA GTT CTG CAG GG & 60 \\
\hline STM0021 & $\mathrm{F}$ & TCT AGA TAA ATC ACA CAC TTC CA & 54 & STM2005 & $\mathrm{R}$ & GTC ATA ACC TTT ACC ATT GCT GG & 60 \\
\hline STM0021 & $\mathrm{R}$ & AAT GTC CCG AGT TGG ATA & 54 & STM2012 & $\mathrm{F}$ & GCG GCC GCT TCT CAG CCA A & 64 \\
\hline STM0028 & $\mathrm{F}$ & CAT AAA TGG TTA TAC ACG CTT TGC & 60 & STM2012 & $\mathrm{R}$ & TCT CGT TCA ATC CAC CAG ATC & 60 \\
\hline STM0028 & $\mathrm{R}$ & TAA TGG AGT TCC TGA AAA GAA AGG & 60 & STM2021 & $\mathrm{F}$ & AAC GTT TGA CTA AGC CCC AGT & 60 \\
\hline STM0030 & $\mathrm{F}$ & AGA GAT CGA TGT AAA ACA CGT & 54 & STM2021 & $\mathrm{R}$ & TTG GTA TGG GGG ATA AAG TTC A & 60 \\
\hline STM0030 & $\mathrm{R}$ & GTG GCA TTT TGA TGG ATT & 54 & STM2023 & $\mathrm{F}$ & TTT TCC GAC GAA ATC CTC C & 60 \\
\hline STM0031 & $\mathrm{F}$ & CAT ACG CAC GCA CGT ACA C & 60 & STM2023 & $\mathrm{R}$ & GAA AAA AGG AGT AGA GGC GGC & 60 \\
\hline STM0031 & $\mathrm{R}$ & TTC AAC CTA TCA TTT TGT GAG TCG & 60 & STM2028 & $\mathrm{F}$ & TCT CAC CAG CCG GAA CAT & 60 \\
\hline STM0037 & $\mathrm{F}$ & AAT TTA ACT TAG AAG ATT AGT CTC & 48 & STM2028 & $\mathrm{R}$ & AAG CTG CGG AAG TGA TTT TG & 60 \\
\hline STM0037 & $\mathrm{R}$ & ATT TGG TTG GGT ATG ATA & 48 & STM2030 & $\mathrm{F}$ & TCT TCC CAA ATC TAG AAT ACA TGC & 59 \\
\hline STM0040 & $\mathrm{F}$ & GCA ATA ATG GCC AAC ACT TC & 58 & STM2030 & $\mathrm{R}$ & AAA GTT AGC ATG GAC AGC ATT TC & 60 \\
\hline STM0040 & $\mathrm{R}$ & TGG GAA ATG TTA GTC AAA AAT AGC & 59 & STM3000 & $\mathrm{F}$ & CAA CCC AAT TTC GAG AGG GAC & 68 \\
\hline STM0050 & $\mathrm{F}$ & TCA GAG GTT TTG TCA CGT T & 54 & STM3000 & $\mathrm{R}$ & TGT CCC AAA ATC GTC TCT GGC & 66 \\
\hline STM0050 & $\mathrm{R}$ & TAT ATG GGA CAC ACG TGC & 53 & STM3010 & $\mathrm{F}$ & TCA TGA GTG CTG GTG CTA CC & 60 \\
\hline STM0052 & $\mathrm{F}$ & TAG GCT CGG GTC ATT ACA ATA A & 59 & STM3010 & $\mathrm{R}$ & GTC TTA ACA GCA AAT CAA CAA TGG & 60 \\
\hline STM0052 & $\mathrm{R}$ & GCT CGC CTG TGT TTG TTG T & 60 & STM3011 & $\mathrm{F}$ & GTG TGG TTG ATT GAT TGA TTT AGC & 60 \\
\hline STM1002 & $\mathrm{F}$ & TAT TCC CCC TTC CTA CTC AA & 56 & STM3011 & $\mathrm{R}$ & GTT TTT AGG CAG TTC TTG GGG & 60 \\
\hline STM1002 & $\mathrm{R}$ & TCT TCC ACA TTC CTA ACC TG & 55 & STM3012 & $\mathrm{F}$ & CAA CTC AAA CCA GAA GGC AAA & 60 \\
\hline STM1004 & $\mathrm{F}$ & ATA TGA AAT TCT CTC GAT GTT TCG & 59 & STM3012 & $\mathrm{R}$ & GAG AAA TGG GCA CAA AAA ACA & 60 \\
\hline STM1004 & $\mathrm{R}$ & TCA GCC CAT AAA XCT TTA GTT ACC T & 58 & STM3016 & $\mathrm{F}$ & TCA GAA CAC CGA ATG GAA AAC & 60 \\
\hline STM1007 & $\mathrm{F}$ & AAT GTC ATT CGT TAC TTC CC & 54 & STM3016 & $\mathrm{R}$ & GCT CCA ACT TAC TGG TCA AAT CC & 60 \\
\hline STM1007 & $\mathrm{R}$ & AGT CAA GGG AGT AAA CAA AG & 51 & STS 1 , STS 2 & $\mathrm{~F}$ & TCT CTT GAC ACG TGT CAC TGA AAC & 60 \\
\hline STM1008 & $\mathrm{F}$ & GTA CAC AGC AAA ATA GCA AG & 56 & STS 1, STS 2 & $\mathrm{R}$ & TCA CCG ATT ACA GTA GGC AAG AGA & 60 \\
\hline STM1008 & $\mathrm{R}$ & TAG ACA CTC TCA CAT CCA CT & 56 & STS 1 , STS 3 & $\mathrm{~F}$ & TCT CTT GAC ACG TGT CAC TGA AAC & 60 \\
\hline STM1016 & $\mathrm{F}$ & TTC TGA TTT CAT GCA TGT TTC C & 60 & STS 1 , STS 3 & $\mathrm{R}$ & TTG CCA TGT GAT GTG TGG TCT AGA A & 60 \\
\hline STM1016 & $\mathrm{R}$ & ATG CTT GCC ATG TGA TGT GT & 60 & STU6SNRN & $\mathrm{F}$ & GAA GTT TTA TCA GAA TCC & 46 \\
\hline STM1019 & $\mathrm{F}$ & TAG ATT TTA TTA TTC CCA ACA AGC A & 58 & STU6SNRN & $\mathrm{R}$ & ATC ACC TCA TCA GCA ATC & 50 \\
\hline STM1019 & $\mathrm{R}$ & CAA CTA CCT ТCT CCC CAC ATA G & 58 & & & & \\
\hline STM1020 & $\mathrm{F}$ & TTC GTT GCT TAC CTA CTA & 47 & & & & ontinued \\
\hline
\end{tabular}


Table 1. Continued.

\begin{tabular}{|c|c|c|c|}
\hline Primer & & Primer sequencez $5^{\prime}-3^{\prime}$ & $\begin{array}{l}\text { Melting } \\
\text { temp }\left({ }^{\circ} \mathrm{C}\right)\end{array}$ \\
\hline STWIN12G & $\mathrm{F}$ & TGT TGA TTG TGG TGA TAA & 46 \\
\hline STWIN12G & $\mathrm{R}$ & TGT TGG ACG TGA CTT GTA & 50 \\
\hline X04753 & $\mathrm{F}$ & GGG TCA AGG GCT AAT ATA AGA & 52 \\
\hline X04753 & $\mathrm{R}$ & ACA AGG ATT AAA AGC ACC TCT & 53 \\
\hline $059-060$ & $\mathrm{~F}$ & GTG ATT GGC AAT CAG ATT GAA A & 54 \\
\hline $059-060$ & $\mathrm{R}$ & GTG TGT GGA CTG TGG AGT GG & 60 \\
\hline DAD340-341 & $\mathrm{F}$ & AAA TCA GAA CTA GTG GGT TCA & 53 \\
\hline DAD340-341 & $\mathrm{R}$ & TGA TAA AAG ATA GAT TCA TAT TGG & 53 \\
\hline STPRINPSG & $\mathrm{F}$ & TGT ACT GGG GAG CCT CAA AG & 58 \\
\hline STPRINPSG & $\mathrm{R}$ & AAT TTT AAC CTC GTG ACA TGG G & 56 \\
\hline POTM 1-2 & $\mathrm{F}$ & AAT AAT ACT GTG ATG CCA CAA TGG & 57 \\
\hline POTM 1-2 & $\mathrm{R}$ & GTG GCA TGT CTT CGA AGG TAC & 59 \\
\hline RV19-20 & $\mathrm{F}$ & AAT AAT ACT GTG ATG CCA CAA TGG & 57 \\
\hline RV19-20 & $\mathrm{R}$ & GTG GCA TGT CTT CGA AGG TAC & 59 \\
\hline RV23-24 & $\mathrm{F}$ & GCT ATG TTA GGG TCA AGG GCT A & 59 \\
\hline RV23-24 & $\mathrm{R}$ & ATA TGG GAT GCT TGG TAT ACC G & 57 \\
\hline RV5-6 & $\mathrm{F}$ & CTT GCA ACT TGT TAG TAC CCC C & 59 \\
\hline RV5-6 & $\mathrm{R}$ & AAA TCC TTT GTG ACC TCC CC & 56 \\
\hline ST13ST & $\mathrm{F}$ & GTG ATT GGC AAT CAG ATT GAA A & 54 \\
\hline ST13ST & $\mathrm{R}$ & GTG TGT GGA CTG TGG AGT GG & 60 \\
\hline STACCAS3 & $\mathrm{F}$ & AAT TCA TGT TTG CGG TAC GTC & 55 \\
\hline STACCAS3 & $\mathrm{R}$ & ATG CAG AAA GAT GTC AAA ATT GA & 53 \\
\hline STGBSS & $\mathrm{F}$ & AAT CGG TGA TAA ATG TGA ATG C & 54 \\
\hline STGBSS & $\mathrm{R}$ & ATG CTT GCC ATG TGA TGT GT & 54 \\
\hline STGBSS & $\mathrm{F}$ & TTA TGA ATC GTG TTA TGG & 46 \\
\hline STGBSS & $\mathrm{R}$ & CAA AAA GGG GAA TCT ACC & 50 \\
\hline STIIKA & $\mathrm{F}$ & TTC GTT GCT TAC CTA CTA & 50 \\
\hline STIIKA & $\mathrm{R}$ & CCC AAG ATT ACC ACA TTC & 50 \\
\hline STINHWI & $\mathrm{F}$ & GGA GTC AAA GTT TGC TCA CAT C & 57 \\
\hline STINHWI & $\mathrm{R}$ & CAC CCT CAA CCC CCA TAT C & 58 \\
\hline
\end{tabular}

zPrimers were developed by Milbourne et al., 1998.

Table 2. Primer sequences for adapters, pre-amplification primers, and selective amplification primers for LI-COR ${ }^{z}$ analysis of the six clonal selections of the potato cultivar Russet Norkotah.

\begin{tabular}{llc}
\hline Oligonucleotide & \multicolumn{1}{c}{ Primer sequence } & $\begin{array}{c}\text { Length } \\
\text { (bp) }\end{array}$ \\
\hline MseI Adapter 1 & 5'-GAC GAT GAG TCC TGA G-3' & 16 \\
MseI Adapter 2 & 3'-TAC TCA GGA CTC AT-5' & 14 \\
PstI Adapter 1 & 5'-GAC TGC GTA GGT GCA-3' & 15 \\
PstI Adapter 2 & 3'-CCT ACG CAG TCT ACG AG- 5' & 17 \\
PstI+0 Pre-Amp Primer & 5'-GAC TGC GTA GGT GCA G-3' & 17 \\
MseI+0 Pre-Amp Primer & 5'-GAT GAG TCC TGA GTA AC-3' & 17 \\
MseI-CNN ${ }^{y}, \mathrm{x}$ & 5'-GAT GAGTCCTGAGTAACNN-3' & 19 \\
PstI-ANN & 5'-GAC TGC GTA GGT GCA GCN N-3' & 19
\end{tabular}

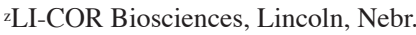

yThe selective nucleotides for $M s e I$ included CGC, CGT, CGA, CGG, CTC, CTT, CTA, CTG, CAC, CAT, CAA, CAG, CCC, CCT, CCG, and CCA.

${ }^{x} \mathrm{NN}$ depicts two nucleotides.

wSelective nucleotides for PstI primers were CAA (IRD dye labeled at 700nm), CGT, and CTT (IRD labeled at $800 \mathrm{~nm}$ ).

AFLP primers pairs used was able to detect reliable polymorphisms among the six 'Russet Norkotah' intraclonal variants, although a total of 3755 AFLP markers were examined. Some polymorphisms were observed, but they were not confirmed with subsequent analysis of the same DNA, and were thus assumed to be amplification artifacts. Out of 2042 bands examined from silver stained gels, $29(1.4 \%)$ produced nonreproducible polymorphisms, whereas $42(2.4 \%)$ of the 1713 bands scored from fluorescent labeled gel images were similarly nonreproducible. Primers analyzed on the LI-COR produced an average of 44 bands per primer, while those that were silver stained produced only 33 .
Eleven primer combinations were not scored due to poor amplification or background on the silver-stained gels. Despite a lack of polymorphisms between the six 'Russet Norkotah' strains, numerous polymorphisms were observed between the clones and ATX85404-8W, resulting in a $14.4 \%$ difference.

Microsatellite analysis. The 79 SSR primer sets were not polymorphic among 'Russet Norkotah' and the strains, as seen with AFLPs. The SSR analysis that was conducted identified 363 bands from 30 primer sets. An additional 49 primer sets were evaluated that were monomorphic among all the 'Russet Norkotah' strains and 'Russet Burbank' or did not amplify clear bands. Seven primer sets did not amplify any bands. Figure 2 is an example of the banding patterns observed when the PCR amplification products were separated on the PAGE system. Twenty-eight primer sets amplified bands that were identical between 'Russet Burbank' and 'Russet Norkotah' (including the line selections). Three primer sets (STM0004, STM0050, and STM2028) amplified bands that appeared to be unique to certain line selections. These three primer sets were then tested in a replicated manner. The replicated analyses suggested that the polymorphic bands observed are spurious (Fig. 3).

\section{Discussion}

Both AFLPand SSR marker systems failed to detect differences between the clones. This inability to detect marker differences between the clones underscores the high degree of genetic similarity among them. The 'Russet Norkotah' clones have repeatedly shown differences in phenotypic traits such as increased yield, larger vine size, and lower nitrogen requirements across multiple environments and years (i.e., they are stable) (Miller et al., 1999; Zvomuya et al., 2002). These differences could be a result of somatic variation. The absence of polymorphisms among the clones suggests that these variations are restricted to very small segments of the genome.

Both silver-stained and fluorescent-labeled gel images revealed a small percentage of nonreproducible polymorphisms. These numbers are within the bounds of error reported in previous studies, suggesting an AFLP error rate of $\approx 2 \%$ (Arens et al., 1998; Winfield et al., 1998). Work conducted by Monte-Corvo et al. (2001) suggested that the difference in the percent of irreproducible polymorphisms between silver staining and other forms of visualization are due to the fact that many of the weak markers responsible for false polymorphisms are not detectible with silver staining. This is supported by the average number of bands per primer in the two systems, with LI-COR primers producing an average of 44 bands per primer, and silver staining only 33 . There is a distinct possibility that technical differences in the detection system could be responsible for the total number of bands detected (Monte-Corvo et al. 2001); however, the detection system may have been confounded by the different combinations of enzymes used in each system. The enzymes used might also have an effect on the number of bands detected, especially since the enzymes are thought to target different areas of the genome. While it is possible that the LI-COR system offers superior resolution, or increased detection of bands with the infrared dye, further research is warranted to determine if this is the only factor affecting the number of bands.

Lack of molecular evidence to differentiate clones may be explained several different ways. First, the PCR products generated from different individuals reflect the length of the amplified region, but not the particular sequence of that region. Therefore, 


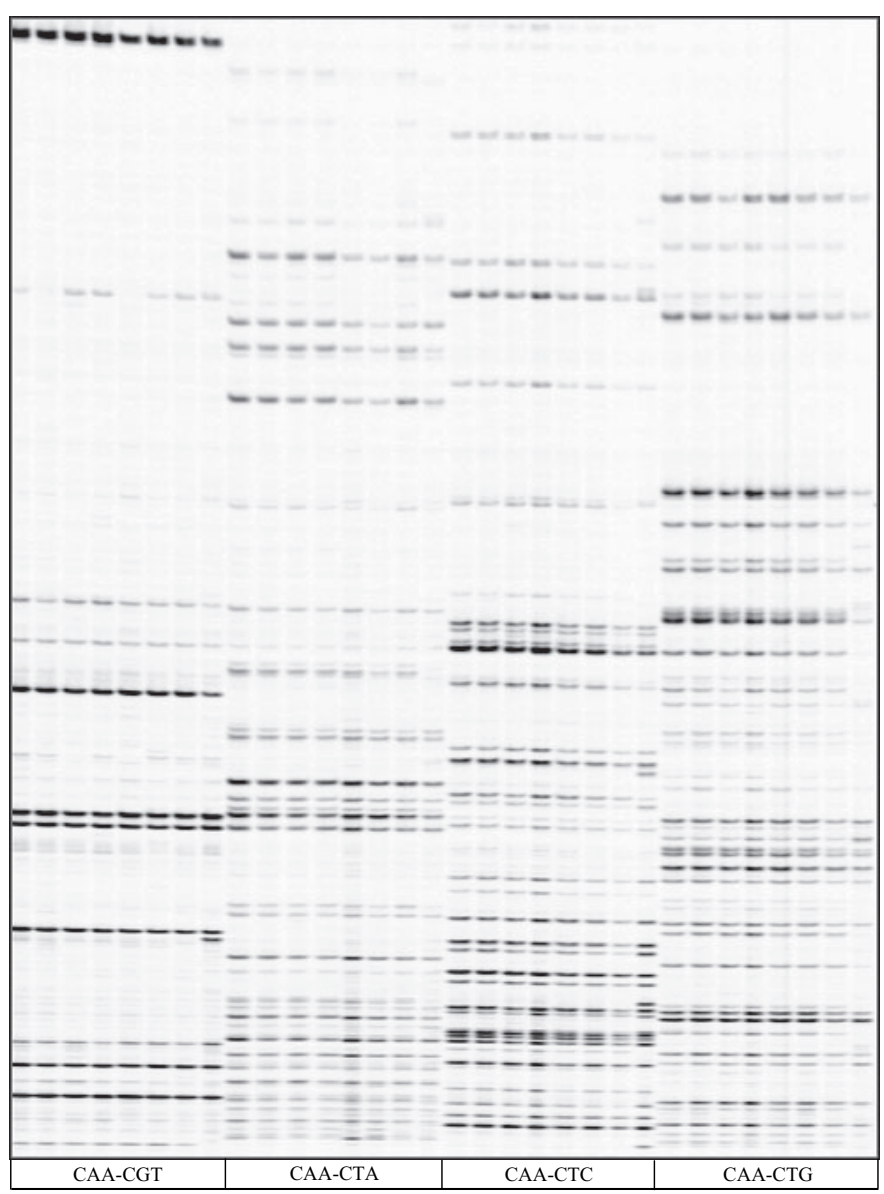

Fig. 1. A representative LI-COR (LI-COR Biosciences, Lincoln, Nebr.) gel with four different MseI/PstI primer pairs is shown above. The potato genotypes, left to right for each primer pair, are CORN 3, TXNS 223, TXNS 112, TXNS 278, TXNS 296, CORN 8, 'Russet Norkotah,' and ATX85404-5W. It is clearly demonstrated that ATX85404-8W has a different amplified fragment length polymorphism pattern from the rest of the genotypes, while 'Russet Norkotah' and its clones are indistinguishable from one another.

a difference in sequence could be present between the clones, but would not be detected if it does not alter the length of the amplified region or the restriction site.

A second possible explanation is differences in methylation. PstI was used in lieu of the EcoRI used in AFLP analysis I beacuse PstI is a methylation-sensitive enzyme, while EcoRI is not. That is, PstI digests DNA only in nonmethylated regions, while $E c o$ RI cuts DNA regardless of the methylation state. Since methylation of DNA is a silencing mechanism, it could explain differences between the clones, and it was potentially useful to look at banding patterns using methylation-sensitive, as well as methylation-insensitive, enzymes in the AFLP analysis. Since, however, the PstI enzyme digests a GC-rich region, and expressed regions frequently are rich in $\mathrm{GC}$ content, nonmethylated expressed regions would likely be preferentially digested and subsequently amplified. Although a methylation-sensitive enzyme was used in this study, unless a restriction site was differentially methylated between standard 'Russet Norkotah' and the clones, methylation differences would not be evident. Since restriction sites represent a very small percentage of the genome, chances are, if there are methylation differences between the clones, they would not occur within these restriction sites.

A third explanation involves the tetraploid nature of potato. Meyer et al. (1998) pointed out the complications of using a

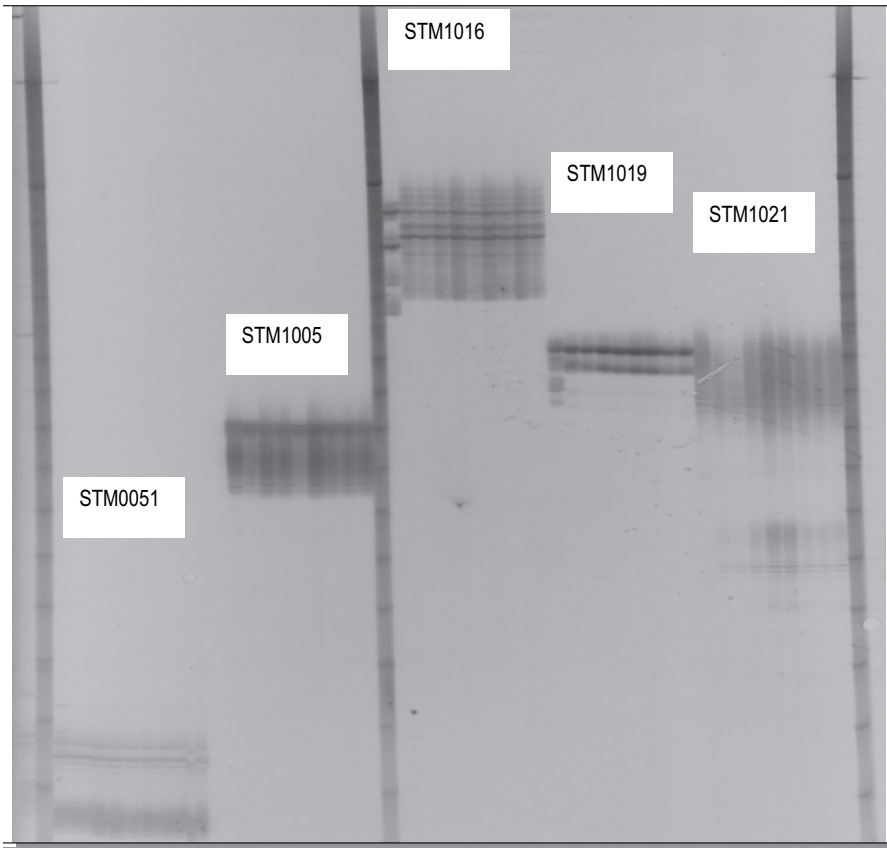

Fig. 2. Example of SSR banding patterns from five primer combinations (STM0051, STM1005, STM1016, STM1019, and STM 1021). Amplified products were separated on polyacrylamide gels and visualized using silver staining. For each of the five primer combinations, lanes (from left to right) are the potato cultivars Russet Burbank, Russet Norkotah, CORN 3, CORN 8, TXNS 102, TXNS 112, TXNS 223, TXNS 278, and TXNS 296. Band sizes were estimated based on 10-bp ladders.

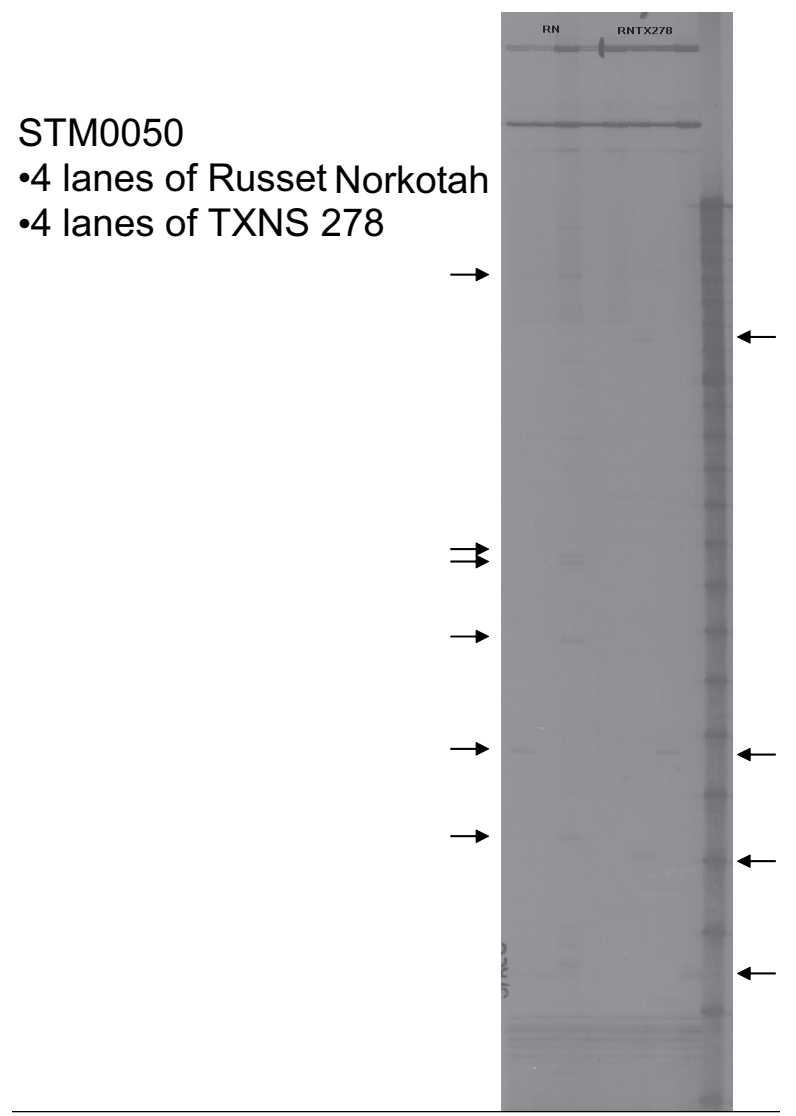

Fig. 3. Example of STM0050 comparing four lanes of the potato cultivars Russet Norkotah and TXNS 278. The arrows indicate the appearance of spurious faint bands. The last lane is a 10 -bp marker. 
tetraploid species in an AFLP analysis. While AFLPs are known for their high multiplex ratios, in tetraploids much of the polymorphism is masked by "dosage." Since AFLPs are frequently dominant markers, due to the presence or absence of a priming site, one would not see differences if this presence or absence was masked by another copy of that site. That is, if there are four copies of a gene, for example AAAa, and it is mutated to AAaa, one would not see this difference. For example, in a diploid species, there are two copies of the hypothetical gene "A." If the genotype is Aa, and the priming site falls in the middle of the "A" form of the gene, a mutation from "Aa" to "aa" would cause a loss of this priming site, and the corresponding loss of a band. In a tetraploid, however, if the genotype is changed from AAAa to Aaaa, the "A" allele would still be present, and a band would still be produced for this allele. Thus, it is possible that one chromosome of the four in 'Russet Norkotah' was mutated in the clones, but since we cannot reliably discriminate between band intensity, it was not detected.

Many studies have been performed on somoclonal variants, and the resulting literature can provide insight into possible explanations for differences in the 'Russet Norkotah' clones. Since the early 1980s, researchers have been trying to determine the underlying cause of tissue culture derived variants, and possible explanations have included everything from gross chromosomal rearrangements to more cryptic changes such as methylation. Frequent explanations are inversions, deletions, translocations, polyploidization, transposon activation, point mutations, and methylation (Larkin and Scowcroft, 1981).

While chromosome breakage is probably accentuated in materials grown under tissue culture conditions, it is still feasible that such breakage occurred with the 'Russet Norkotah' clones. When such breakage occurs, there is a great potential for loss of genetic material. Furthermore, it could affect genes in which a break occurs, as well as neighboring genes under the control of the same promoter. In addition, translocation of a gene can have a positional effect, with one possible result the silencing of a previously expressed gene. Should a dominant allele be "turned off" the phenotype of the recessive allele will be expressed, thus leading to an altered phenotype (Larkin and Scowcroft, 1981). While it may seem unlikely that screening the genotypes with 112 AFLP primers would miss such an event, it is, in fact, very plausible. Unless a deletion occurs within two restriction sites, it would go undetected. Furthermore, an inversion not altering the length of an amplified fragment would also go undetected.

Clearly, there are stable genetic differences between 'Russet Norkotah' and its strains, but the right marker system to uncover these differences is not likely to be based on fragment variation as detected by AFLPs, SSRs, or other anonymous molecular markers. Differences among clones are probably due to point mutations that might be better identified by techniques such as single-strand confirmation polymorphism (SSCP), temperature gradient gel electrophoresis (TGGE), or denaturation gradient gel electrophoresis (DGGE). The usefulness of these techniques in discriminating between intraclonal variants has been under investigation by Monte-Corvo et al. (2001). Another possible technique to detect differences between these and other intraclonal variants would be to test the relatively new high-throughput "gene chip" technology. The elucidation of differential expression of the genes in 'Russet Norkotah' could ultimately allow differentiation of clones and, in turn, determine the genes responsible for their variant phenotypes.

\section{Literature Cited}

Arens, P., H. Coops, J. Jansen, and B. Vosman. 1998. Molecular genetic analysis of black poplar (Populus nigra L.) along Dutch rivers. Mol. Ecol. 7:11-18.

Cabrita, L.F., U. Aksoy, S. Hepaksoy, and J.M. Leitao. 2001. Suitability of isozyme, RAPD, and AFLP markers to assess genetic differences and relatedness among fig (Ficus carica L.) clones. Sci. Hort. 87:261-273

Fritz, A.K., S. Caldwell, and W.D. Worall. 1999. Molecular mapping of Russian wheat aphid resistance from triticale accession PI 386156. Crop Sci. 39:1707-1710.

Fulton T., C. Juliapark, and S. Tanksley. 1995. Miniprep protocol for extraction of DNA from tomato and other herbaceous plants. Mol. Biol. Rpt. 13:207-209.

Johansen, R. H., B. Farnsworth, D. C. Nelson, G. A. Secor, N. Gudmestad, and P. H. Orr. 1988. Russet Norkotah: A new russet-skinned potato cultivar with wide adaptation. Amer. Potato J. 65:597-604.

Kardolus, J., J.H.J. van Eck, and R.G. van den Berg. 1998. The potential of AFLPs in biosystematics: A first application in Solanum taxonomy (Solanaceae). Plant Systematic Evol. 210:87-103.

Kim, J.H., H. Joung, and Y.P. Lim. 1998. Estimation of genetic variation and relationship in potato (Solanum tuberosum) cultivars using AFLP markers. Amer. J. Potato Res. 75:107-112.

Klein, P.E., R.R. Klein, S.W. Cartinhour, P.E. Ulanch, J. Dong, J.A. Obert, D.T. Morishige, S.D. Schlueter, K.L. Childs, M. Ale, and J.E. Mullet. 2000. A high-throughput AFLP-based method for constructing integrated genetic and physical maps: Progress toward a sorghum genome map. Genome Res. 10:789-807.

Larkin, P.J. and W.R. Scowcroft. 1981. Somoclonal variation-A novel source of variability from cell cultures for plant improvement. Theor. Appl. Genet. 60: 197-214.

McGregor, C.E., C.A. Lambert, M.M. Greyling, J.H. Louw, and L. Warnich. 2000. A comparative assessment of DNA fingerprinting techniques (RAPD, ISSR, AFLP, and SSR) in tetraploid potato (Solanum tuberosum L.) germplasm. Euphytica 113:135-144.

Meksem, K., D. Leister, J. Peleman, M. Zabeau, F. Salamini, and C. Gebhardt. 1995. A high-resolution map of the vicinity of the R1 locus on chromosome $\mathrm{V}$ of potato based on RFLP and AFLP markers. Mol. Gen. Genet. 249:74-81.

Menz, M.A., R.R. Klein, J.E. Mullet, J.A. Obert, N.C. Unruh, and P.E. Klein. 2002. A high-density genetic map of Sorghum bicolor (L.) Moench based on 2926 AFLP, RFLP, and SSR markers. Plant Mol. Biol. 48:483-499.

Meyer, R.C., D. Milbourne, C.A. Hackett, J.E. Bradshaw, J.W. McNichol, and R. Waugh. 1998. Linkage analysis in tetraploid potato and association of markers with quantitative resistance to late blight (Phytopthora infestans). Mol. Gen. Genet. 259:150-160.

Milbourne, D., R. Meyer, J.E. Bradshaw, E. Baird, N. Bonar, J. Provan, W. Powell, and R. Waugh. 1997. Comparison of PCR-based marker systems for the analysis of genetic relationships in cultivated potato. Mol. Breed. 3:127-136.

Milbourne, D., R.C. Meyer, A.J. Collins, L.D. Ramsay, C. Gebhardt, and R. Waugh. 1998. Isolation, characterization and mapping of simple sequence repeat loci in potato. Mol. Gen. Genet. 259:233-245.

Miller, J.C. 1954. Selection of desirable somatic mutations: A means of potato improvement. Amer. Potato J. 31:358-359.

Miller, J.C., Jr., D.C. Scheuring, J.P. Miller, and G.C.J. Fernandez. 1999. Selection, evaluation, and identification of improved Russet Norkotah strains. Amer. J. Potato Res. 76:161-167.

Miller, J.C., Jr., D.G. Smallwood, J.P. Miller, and G.C.J. Fernandez. 1995. Norgold Russet and Norgold Russet M: Additional evidence for genetic dissimilarity. Amer. Potato J. 72:273-286.

Miller, J.C., Jr., G.C. Tai, B. Ouellette, and J.P. Miller. 2004 . Discriminating Russet Norkotah intraclonal selections using canonical and cluster analysis. Amer. J. Potato Res. 81:31-35. 
Monte-Corvo, L., L. Goulao, and C. Oliveira. 2001. ISSR analysis of cultivars of pear and suitability of molecular markers for clone discrimination. J. Amer. Soc. Hort. Sci. 126:517-522.

National Potato Council. 1998. Potato statistical yearbook. Natl. Potato Council, Greenwood Village, Colo.

National Potato Council. 2000. 2000-2001 Potato statistical yearbook. Natl. Potato Council, Greenwood Village, Colo.

National Potato Council. 2002. 2002-2003 Potato statistical yearbook. Natl. Potato Council, Washington, D.C.

National Potato Council. 2003. 2003-2004 Potato statistical yearbook. Natl. Potato Council, Washington, D.C.

Provan, J., W. Powell, and R. Waugh. 1996. Microsatellite analysis of relationships within cultivated potato (Solanum tuberosum). Theor. Appl. Genet. 92:1078-1084.

Raker, C. and D.M. Spooner. 2002. The Chilean tetraploid cultivated potato, Solanum tuberosum, is distinct from the Andean populations; Microsatellite data. Crop Sci. 45:1451-1458.

Renganayaki, K., J.C. Read, and A.K. Fritz. 2001. Genetic diversity among Texas bluegrass genotypes (Poa arachnifera Torr.) revealed by AFLP and RAPD markers. Theor. Appl. Genet. 102:1037-1045.

Sambrook, J., E.F. Frisch, and T. Maniatis. 1989. Molecular cloning: A laboratory manual, 2nd ed. Cold Spring Harbor Laboratory Press, New York.

Schneider, K. and D. Douches. 1997. Assessment of PCR-based simple sequence repeats to fingerprint North American potato cultivars. Amer. Potato J. 74:149-160.

Vignani, R., J.E. Bowers, and C.P. Meredith. 1996. Microsatellite DNA polymorphism analysis of clones of Vitis vinifera 'Sangiovese.' Sci. Hort. 65:163-169.

Winfield, M.O., G.M. Arnold, F. Cooper, M. LeRay, J. White, A. Karp, and K.J. Edwards. 1998. A study of genetic diversity in Poplus nigra subsp. betulifolia in the Upper Severn area of the UK using AFLP markers. Mol. Ecol. 7:3-10.

Zvomuya, F., C.J. Rosen, and J.C. Miller, Jr., 2002. Response of Russet Norkotah clonal selections to nitrogen fertilization. Amer. J. Potato Res. 79:231-239. 